\title{
Jogo de Tabuleiro "Programming" - uma nova estratégia pedagógica para o ensino e a aprendizagem de Algoritmo
} Board Game "Programming" - a new pedagogical strategy for the teaching and learning of Algorithm

\author{
Marcos Gabriel Rodrigues Melo, Clara Larissa Duarte Trajano Dias, Yasmim da Silva \\ Sales, Eduardo Garcia Wanderley \& Renata Garcia Wanderley
}

\begin{abstract}
Algoritmos, design, jogo, aprendizagem
A disciplina "Algoritmos" é componente do currículo do curso técnico em Informática do IFPE - Garanhuns. Nela, os estudantes possuem o primeiro contato com os conceitos de lógica e linguagem de programação. Apesar disso, um estudo realizado pela coordenação de curso aponta que nos últimos anos a taxa de reprovação nesta tem sido superior a $50 \%$. Diante dessa problemática, observa-se a necessidade da criação e inserção de novas estratégias didático-pedagógicas no ensino e a aprendizagem da disciplina em questão, e os jogos são uma dessas possíveis estratégias. Assim sendo, essa pesquisa busca construir um jogo de tabuleiro para o ensino de Algoritmos. Para tanto, desenvolveuse uma pesquisa projetual fundamentada em pesquisas bibliográficas nas áreas da Educação, Ciência da Computação e Design da Informação. Como resultado final desta pesquisa foi desenvolvido o "Programming", um jogo de tabuleiro em trilha com enfoque no ensino de algoritmos, lógica e lógica de programação. A versão atual do jogo conta com 3 modalidades, sendo duas baseadas em linguagens de programação (Portugol e Java) e outra que aborda apenas o raciocínio lógico-abstrato (modalidade Arbitrária).
\end{abstract}

\section{Algorithms, design, game, learning}

The subject "Algorithms" is part of the syllabus of the technical course in information technology of IFPE Garanhuns. In it, students have the first contact with the concepts of logic and programming language. Despite this, a study carried out by course coordination points out that in recent years the reprobation rate in this one has been higher than $50 \%$. Facing this problem, it is necessary to create and insert new didactic-pedagogical strategies for the teaching and learning of the subject in question and the games are one of these possible strategies. Therefore, this research seeks to construct a board game for the teaching of Algorithms. For this, a research project was developed based on bibliographic research in the areas of Education, Computer Science and Information Design. As a final result of this research was developed "Programming", a track board game with a focus on teaching algorithms, logic and programming logic. The current version of the game has 3 modes, two of them based on programming languages (Portugol and Java) and another that deals only with logical-abstract reasoning (Arbitrária mode).

\section{Introdução}

A programação de computadores revela-se um requisito indispensável no mundo informacional no qual estamos inseridos. Ter um bom conhecimento ou domínio do tema é um diferencial necessário, uma vez que caminhamos para contextos cada vez mais automatizados e intermediados por programas em máquinas ou computadores (Rushkoff, 2012).

Nessa atual configuração, outros aspectos passaram a ter relevância na sociedade: valorizou-se o conhecimento; a riqueza dos países passou a ser medida pelo acesso à tecnologia e sua capacidade de desenvolvimento na área; a informação e as práticas relacionadas a ela se tornaram o principal setor da economia. [...] Com o uso do computador, os serviços foram agilizados e facilitados, houve uma redução da mão-de-obra em ocupações que substituíram o trabalho humano, mas que abriu portas para novas ocupações especializadas no ramo da informática (programadores, webdesigners, administradores de rede) e das comunicações (Kohn \& Moraes, p. 2, 2007).

Como consequência direta, a demanda por programadores aumenta a cada ano no

Anais do 9 CIDI e 9 CONGIC

Luciane Maria Fadel, Carla Spinillo, Anderson Horta, Cristina Portugal (orgs.)

Sociedade Brasileira de Design da Informação - SBDI Belo Horizonte | Brasil | 2019

ISBN 978-85-212-1728-2
Proceedings of the 9th CIDI and 9th CONGIC

Luciane Maria Fadel, Carla Spinillo, Anderson Horta, Cristina Portugal (orgs.)

Sociedade Brasileira de Design da Informação - SBDI Belo Horizonte | Brazil | 2019

ISBN 978-85-212-1728-2 
mercado. Entretanto, o que se observa na realidade é a escassez cada vez mais crescente desse tipo de profissional. Segundo Piva Jr. e Freitas (2010), as possíveis explicações deste processo estão nas próprias instituições de ensino. Uma quantidade substancial dos alunos nos cursos superiores de Computação não consegue desenvolver de forma eficaz a competência de abstração de problemas.

Nos cursos da área de Informática e Computação, a disciplina de Algoritmos exerce um papel fundamental no desenvolvimento dos discentes na programação, já que é uma das primeiras a introduzir conceitos como raciocínio lógico, matemática, interpretação e abstração na resolução de problemas de maneira sistemática (Silva, 2017). Entretanto, por tratar de aspectos cognitivos que exigem uma alta percepção do abstrato, nota-se um baixo desempenho dos discentes. Segundo dados da coordenação de curso do Instituto Federal de Pernambuco, Campus Garanhuns, a disciplina, na modalidade de ensino técnico em Informática integrada, já chegou a alcançar uma taxa de reprovação de 60,6\% no ano de 2015. Apesar deste índice ter reduzido nos últimos anos, a disciplina, ainda nesta modalidade de ensino, continua atingindo notas anuais críticas, de aproximadamente 4,1 (censo de 2017).

Nesse sentido, a demanda pelo desenvolvimento de meios didático-pedagógicos para o ensino da programação tem sido cada vez mais elevada, principalmente aquelas que tomam como base a interação, a dinâmica e um design funcional e visualmente atrativo.

Diante do exposto, esta pesquisa objetiva a construção de um jogo de tabuleiro para o auxílio ao ensino de Algoritmos. Com isso, buscou-se: (1) identificar as informações (conhecimentos e ações) indispensáveis à temática de algoritmos e (2) construir um sistema de trabalho com tais informações para o aprendizado do conteúdo da disciplina de uma forma mais eficaz e efetiva.

Com isso, surge o jogo "Programming", jogo desenvolvido por discentes do Ensino Médio integrado ao curso técnico em Informática do IFPE Campus Garanhuns, que busca difundir o ensino da programação da forma mais dinâmica e interativa possível a partir deste material didático-pedagógico aos professores. Um dos principais resultados que se busca alcançar com o jogo é a facilitação e fluidez da aprendizagem, a identificação e a familiarização dos discentes em relação à programação. $\mathrm{O}$ jogo tem o intuito de ser mais uma ferramenta de apoio ao ensino e à aprendizagem, oferecendo mais possibilidades aos professores e mais autonomia aos discentes.

Para tanto, desenvolveu-se uma pesquisa projetual que culminou com a construção de um objeto prototipado (o jogo de tabuleiro). Como método de abordagem, implantou-se o dedutivo, com a formação do conhecimento baseado em informações gerais de múltiplas áreas aplicadas em um contexto pontual. Dois métodos de procedimento foram trabalhados: o funcionalista e o estruturalista. Foi gerado um modelo de expressão (o jogo), através da desconstrução e reconstrução do objeto de estudo, baseando-se na definição e análise da funcionalidade de cada unidade para construção do todo.

A pesquisa bibliográfica estruturou toda a base teórica que orientou a execução da pesquisa projetual. Os conhecimentos das referências bibliográficas das áreas já citadas anteriormente foram analisados de forma crítica e apontaram algumas diretrizes técnicas, estéticas e simbólicas aplicadas de forma experimental na construção dos primeiros protótipos.

\section{Fundamentação teórica}

Os jogos são uma das formas mais populares de entretenimento e estão presentes desde a infância. Sua história, sendo objeto intermediador de conhecimento, remonta ao século XVI, quando os humanistas passaram a perceber seu valor educativo (Cunha, 2012). Continuam se manifestando de forma exponencial como poderosas ferramentas na potencialização do processo de aprendizagem (Nunes \& Ziede, 2016)

Nas instituições, a concepção do ensino direcionado pelo interesse do discente passou a 
ser um dos maiores desafios dentro da docência. Nesse meio, os jogos surgem como um instrumento motivador, à medida que estimulam e desafiam os estudantes na resolução de problemas. Os jogos direcionam as atividades de uma forma diferenciada das metodologias tradicionais de ensino (Cunha, 2012).

A dinâmica estabelecida entre discente-docente também permite ao professor análises e questionamentos mais profundos acerca do desenvolvimento e forma do raciocínio dos alunos. Nesse sentido, o docente terá condições de identificar pontualmente as principais deficiências dos alunos e trabalhá-las a fim de saná-las (Piva Jr. \& Freitas, 2010), processo que seria praticamente inatingível em abordagens tradicionais de ensino, visto o curto período de tempo e as dimensões volumosas de conteúdos e turmas.

Sob outra perspectiva, o fato dos jogos de tabuleiro envolverem de forma maciça diversas informações visuais para embasar suas ações, eles demandam a orientação do design e do design da informação na estruturação de seu processo de ideação lógica e construção física. $O$ design trabalha de forma expressiva com a estética e a simbologia, qualificações indispensáveis para qualquer processo de comunicação e produção. O design da informação, por sua vez, otimiza os processos de aquisição e gerenciamento da informação visual. Por conseguinte, os jogos de tabuleiro, com seu caráter lúdico, pedagógico, engajador e principalmente comunicacional, necessitam destas preocupações e qualificações para sua eficácia geral.

Tais fatores expressam sua relevância ainda mais quando o tema a ser tratado é a disciplina de Algoritmos. Uma das principais deficiências dos estudantes quando tratamos do pensamento sistemático e abstração é a interpretação inadequada ou insuficiente dos problemas (Piva Jr. \& Freitas, 2010). Sendo assim, o design atua sendo um verdadeiro instrumento facilitador na interpretação mais íntegra e clara dos problemas, contribuindo na construção de sua possível resolução.

\section{0 jogo}

O jogo "Programming" é um jogo de tabuleiro que utiliza a dinâmica desse tipo de jogo com o tema. Sua construção foi iniciada em 2017, tomando como forma de auxílio teórico a disciplina de Educação Física I, no curso de Informática integrado ao Ensino Médio do IFPE Garanhuns. Nela, os discentes tiveram contato com o conteúdo "jogos de tabuleiro", além de suas aplicações enquanto instrumentos didáticos e estimulantes. Com a reconstrução gradativa do material produzido, deu-se início suas primeiras aplicações com o público, as quais obteve-se um retorno positivo.

O jogo poderá ser usado tanto em cursos técnicos e superiores da área de Informática quanto em escolas de Ensino Fundamental 2 e Ensino Médio, uma vez que sua versão atual está subdividida em 3 modalidades: duas trabalham com conceitos técnicos e linguagem de programação (a pseudolinguagem Portugol e a linguagem Java) e uma trabalha somente com lógica, abstração e sistematização (modalidade nomeada "Arbitrária"). Busca-se, portanto, além da reversão do quadro crítico da disciplina nos cursos de Informática, atuar como um instrumento de fomento ao exercício do pensamento sistemático, lógico e computacional.

Como cada linguagem de programação possui sintaxes e comandos distintos, o tabuleiro foi projetado para ser flexível, com suas casas de ação removíveis (Figura 1), permitindo assim sua adaptação de acordo com o conteúdo dado pelo docente. Uma das grandes vantagens do jogo, além da possibilidade de adaptação, é a sua capacidade de promover a interação entre os jogadores, já que estes são estimulados frequentemente a desafiar seus adversários, a resolverem dos Desafios e a estarem sempre atentos aos movimentos um dos outros. 
Melo, M. G. R., Dias, C. L. D. T., Sales, Y. A. S., Wanderley, E. G. \& Wanderley, R. G.. | Jogo de

Figura 1: Programming "desmontado". (usado com a permissão de Melo e Dias)

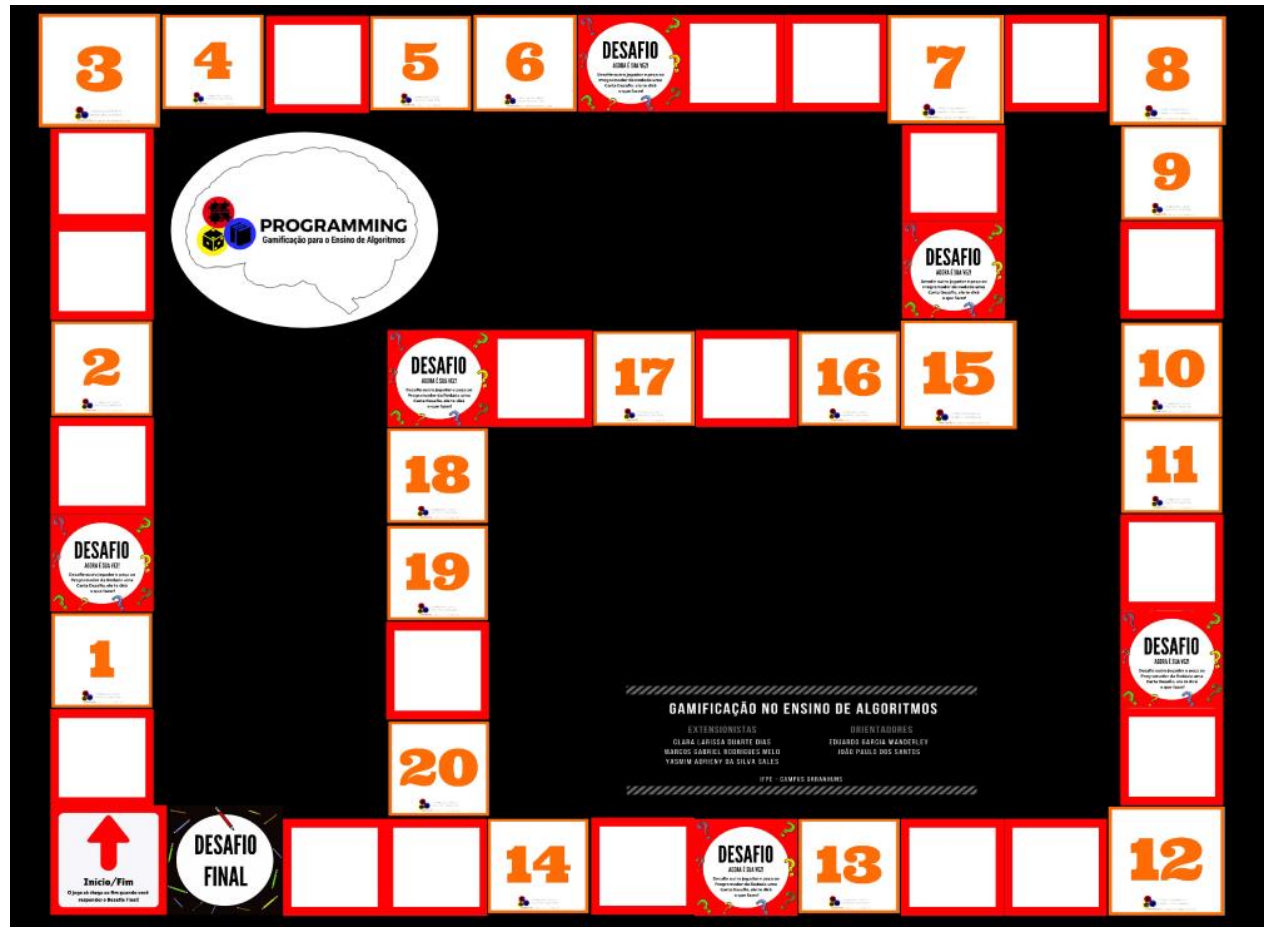

\section{A dinâmica do jogo}

Para construir o conhecimento gradativamente, foi escolhido como estratégia o jogo de trilha. Assim sendo, o principal objetivo do jogador é percorrer todo o caminho demarcado, passando pelos pelas casas de ação e respondendo os problemas propostos, até chegar à penúltima casa, onde obrigatoriamente deverá realizar o desafio final e respondê-lo corretamente para se tornar o vencedor, como observado na Figura 2. Os números marcados no tabuleiro correspondem às casas de ação que deverão ser colocadas em cada local, de acordo com a modalidade escolhida.

Figura 2: Programming montado em sua modalidade "Abitrária". (usado com a permissão de Melo e Dias)

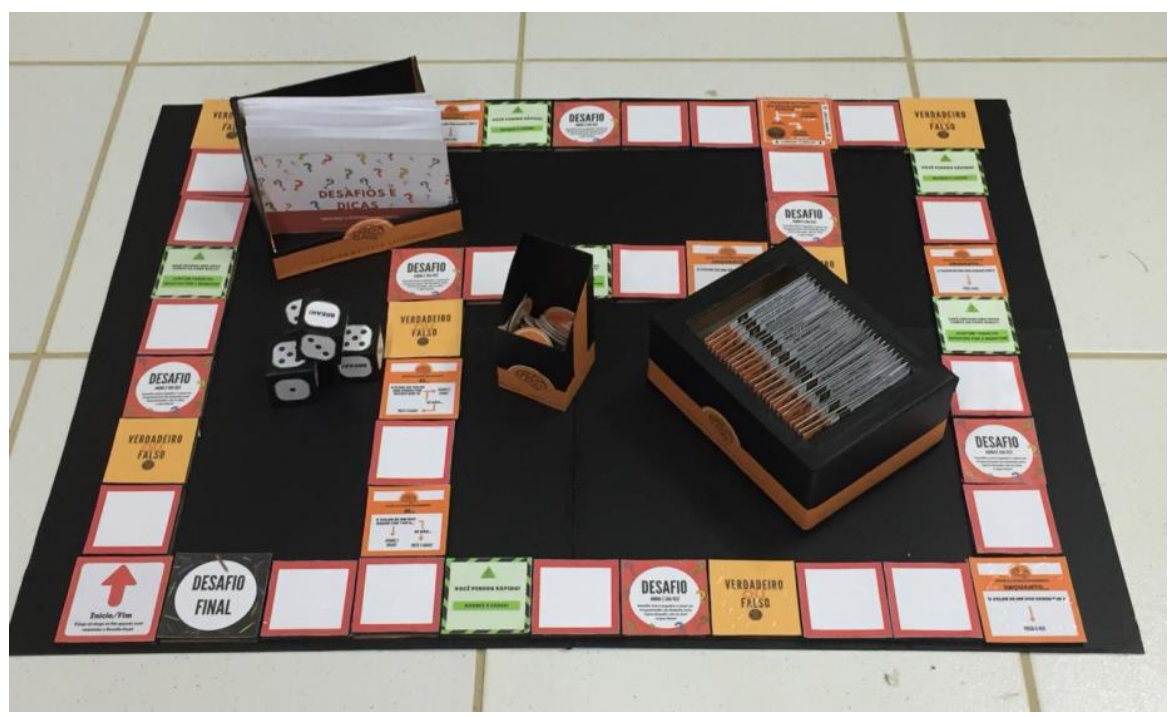


Há um participante central, o Programador da Rodada (papel atribuído geralmente ao professor). Ele é responsável pela administração da mesa de jogo e pela correção das respostas dadas pelos demais participantes, por isso deve ter um conhecimento sólido dos conceitos tratados na modalidade e das regras de funcionamento do jogo. Como forma de apoio, todas as respostas corretas das cartas de Verdadeiro ou Falso estão disponibilizadas junto ao manual do jogo.

O Programming conta com várias casas de ação que estão espalhadas pelo tabuleiro de acordo com a modalidade escolhida. Assim, a partir de sua posição no tabuleiro, o jogador poderá realizar três tipos de ação durante a partida: (1) resolução de problemas via algoritmo através das casas Desafio e Desafio Final (Figura 3), (2) prática de conhecimentos relativos aos conteúdos tratados na modalidade por meio das casas Verdadeiro ou Falso (Figura 4) e (3) visualização, direta ou indireta, das sintaxes de estruturas utilizadas na programação, por intermédio dos obstáculos que compõem a trilha (Figura 5).

Figura 3: Exemplo de carta Desafio. Modalidade Arbitrária. (usado com a permissão de Melo e Dias)

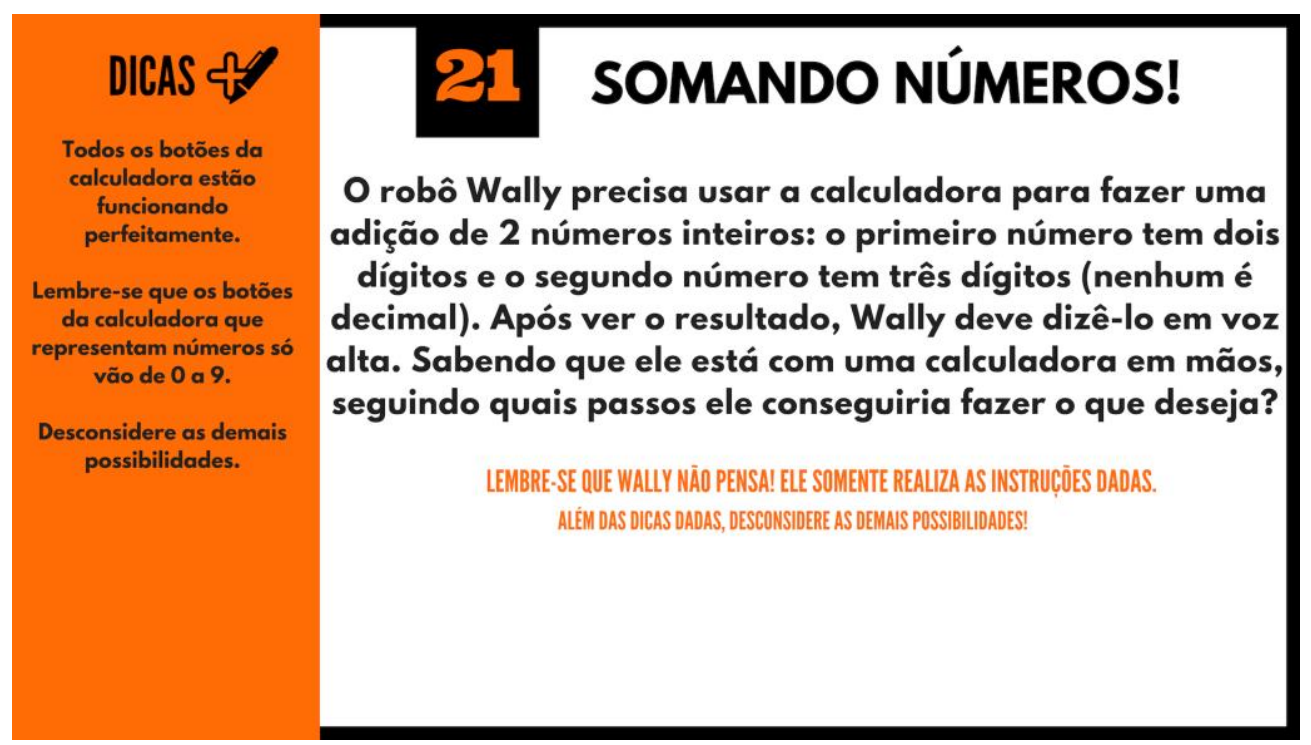

Figura 4: Exemplo das cartas de Verdadeiro ou Falso nas modalidades Arbitrária (A) e Java (B). (usado com a permissão de Melo e Dias)

\section{VERDADEIRO OU FALSO}

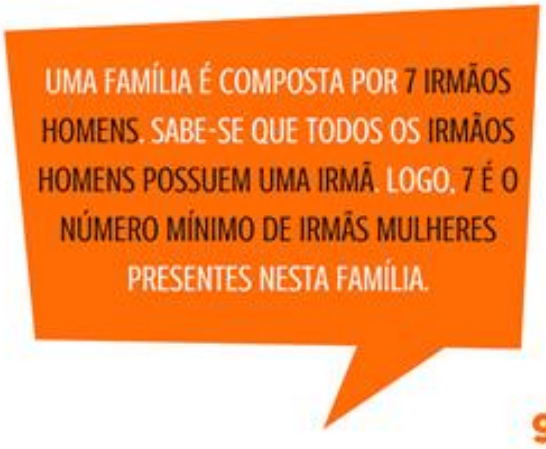

(A)
TRUE OR FALSE

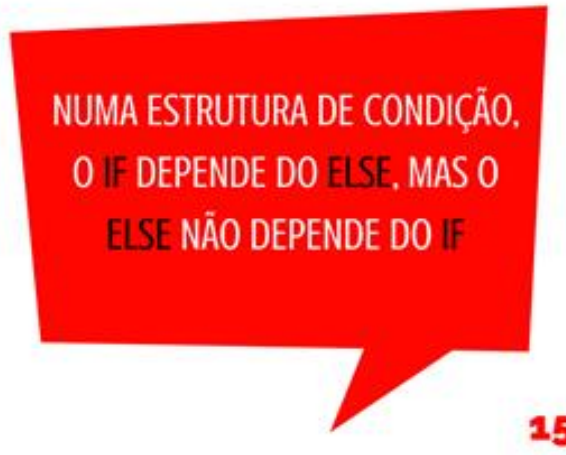

(B) 
Melo, M. G. R., Dias, C. L. D. T., Sales, Y. A. S., Wanderley, E. G. \& Wanderley, R. G.. | Jogo de

Tabuleiro "Programming" - uma nova estratégia pedagógica para o ensino e a aprendizagem de

Figura 5: Exemplo de um dos obstáculos presentes no jogo. (usado com a permissão de Melo e Dias)

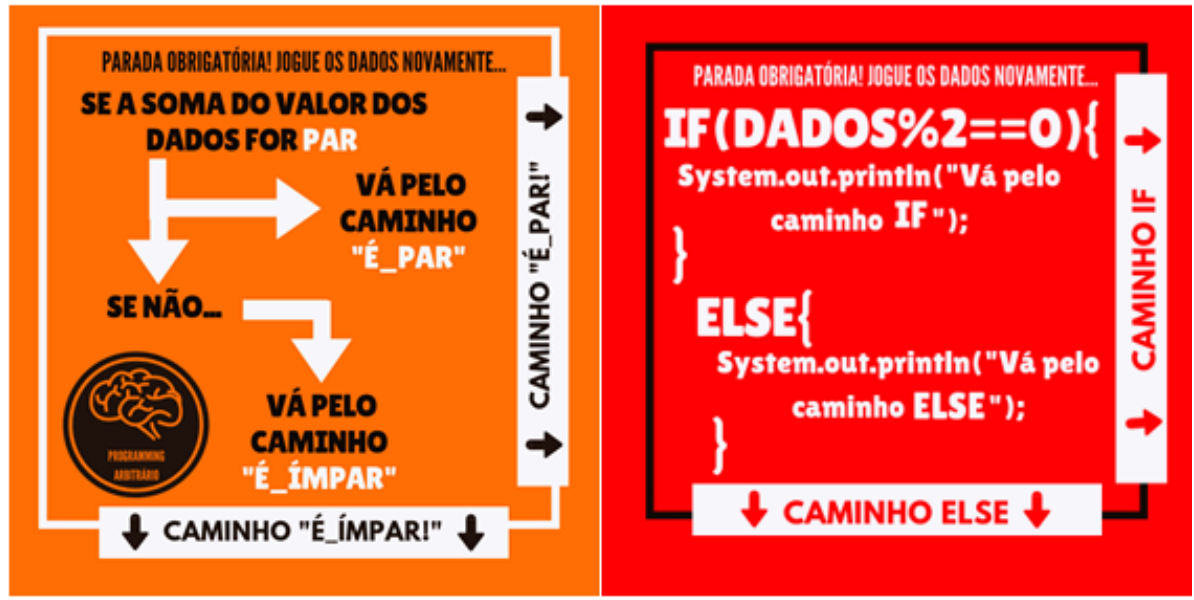

(A)

(B)

Figura 4: Exemplo das cartas de Verdadeiro ou Falso nas modalidades Arbitrária (A) e Java

As regras completas e o conjunto total de Casas de Ação e Cartas do jogo estão disponíveis no site do projeto (seligaaeifpe.wixsite.com/seligaae-ifpe2018).

\section{Avaliações do jogo}

A primeira intervenção feita com o material foi realizada em 2018, durante a Semana de Informática do IFPE - Garanhuns, evento aberto ao público geral. O Programming foi jogado por cerca de cinquenta participantes, divididos em rodadas com grupos de seis integrantes. Posteriormente, já aperfeiçoado, o jogo foi aplicado novamente, ainda em 2018, em mais três momentos: um com estudantes secundaristas, um com estudantes que já concluíram o Ensino Médio (sendo estes dois realizados durante a Semana Nacional de Ciência e Tecnologia do Campus), e um com estudantes dos anos finais do Ensino Fundamental 2. Após o jogo, todos participantes (totalizando cerca de 100 pessoas), em todas as aplicações, foram convidados a responderem um questionário de avaliação como forma de feedback.

Os resultados obtidos com a primeira aplicação foram bastante positivos. Cerca de $91 \%$ dos participantes avaliaram o jogo com notas superiores a oito, e 95,5\% deles afirmaram que jogariam o jogo novamente dentro e/ou fora do Campus. Ao serem questionados sobre como o jogo poderia ser implementado no processo pedagógico, $63,3 \%$ do público acredita que o conjunto formado pelo Programming e lista de exercícios poderá contribuir efetivamente em seus desenvolvimentos, enquanto $31,8 \%$ alega que só o uso do jogo já seria satisfatório. Mais de $90 \%$ avaliaram o tempo do jogo suficiente e julgaram a dificuldade das perguntas das cartas verdadeiro ou falso e desafios adequadas.

No segundo feedback, com o jogo já aperfeiçoado, todos os estudantes (100\% do público) deram notas superiores a oito ao jogo, com aproximadamente $60 \%$ das avaliações sendo nota máxima (dez). Além disso, 100\% dos estudantes também afirmam que jogariam o jogo novamente e o recomendariam/usariam em outras ocasiões. Esta mesma taxa também concordou que o jogo tem capacidade e potencial de auxiliar no desenvolvimento do pensamento sistemático.

\section{Considerações finais}

Na busca por um material de caráter facilitador do ensino de programação e lógica de 
Melo, M. G. R., Dias, C. L. D. T., Sales, Y. A. S., Wanderley, E. G. \& Wanderley, R. G.. | Jogo de Tabuleiro "Programming" - uma nova estratégia pedagógica para o ensino e a aprendizagem de

programação, apresentou-se como resultado final desta pesquisa o jogo de tabuleiro "Programming". Ao propor sua implementação no processo de ensino-aprendizagem, não se visa à substituição das aulas convencionais, mas à inserção de metodologias alternativas dentro do leque no qual os professores têm à disposição.

A implementação de mecanismos de jogos no processo de ensino e aprendizagem envolve não apenas o caráter emocional, mas também o domínio técnico. Seu caráter lúdico e seu processo de trabalho técnico facilitam a imersão no cenário do tema e ajudam com a fixação dos conteúdos e com o desenvolvimento em diversas habilidades, como pensamento sistemático e o raciocínio lógico.

Tomadas as bases teóricas importantes da área de pedagogia, computação e design, construiu-se o conhecimento-foco através de um material físico e de um processo didático simbólico e estimulante. Assim, o jogo trabalha por meio de um caminho sistemático e hierárquico, de ações, pensamentos e decisões e de projeto gráfico informativo.

Com o teste, observou-se que o jogo é dinâmico e lúdico, permitindo uma abordagem interessante e eficaz com os conhecimentos necessários à disciplina de Algoritmos. O design da informação ajudou a organizar e distribuir as ideias que constroem o conhecimento, bem como apresentá-la de forma visual gráfica.

A partir dos resultados já obtidos, é permitida a projeção de expectativas relacionadas à diminuição das taxas de reprovação e evasão dos alunos na disciplina de Algoritmos, além da efetividade da plataforma desenvolvida como uma eficaz alternativa aos professores que buscam por tecnologias educacionais mais dinâmicas. Contudo, ainda se faz necessário o desenvolvimento e inclusão de ainda mais alternativas pedagógicas que permitam mais autonomia aos discentes e incrementem esse leque dos docentes. Por isso, como perspectiva futura, além da continuidade das intervenções feitas com o jogo nas turmas de Informática, busca-se a produção da plataforma gamificada "Se Liga!", que tem como finalidade, em conjunto como o Programming, acompanhar os alunos durante toda a trilha de aprendizagem, contendo três principais vertentes: Teorização, Exemplificação e Prática.

\section{Agradecimento}

Nossos agradecimentos ao IFPE (Pibex) pelo apoio financeiro necessário para a construção dos materiais.

\section{Referências}

Cunha, M. B. (2012). Jogos no Ensino de Química: Considerações Teóricas para sua Utilização em Sala de Aula. São Paulo: Química Nova na Escola v. 34, n. 2.

Kohn, K., \& Moraes, C. H. (2007). O impacto das novas tecnologias na sociedade: conceitos e características da Sociedade da Informação e da Sociedade Digital. Santos: Anais do XXX Intercom.

Nunes, M. R., \& Ziede, M. K. L. (2016). A Construção de games pelos professores das séries iniciais da rede pública do município de Caçador. Lages: Anais do I Edupala.

Piva Jr., D., \& Freitas, R. L. (2010). Estratégias para melhorar os processos de abstração na disciplina de Algoritmos. João Pessoa: Anais do XXI SBIE.

Rushkoff, D. (2012). As 10 Questões Essenciais da Era Digital. São Paulo: Saraiva.

Silva, L. S., \& et al. (2017). Aprendizagem Móvel de Conceitos Introdutórios de Programação: Uma Revisão Sistemática dos Jogos. Fortaleza: Anais do XXII TISE. 
Melo, M. G. R., Dias, C. L. D. T., Sales, Y. A. S., Wanderley, E. G. \& Wanderley, R. G.. | Jogo de Tabuleiro "Programming" - uma nova estratégia pedagógica para o ensino e a aprendizagem de

\section{Sobre os autores}

Marcos Gabriel Rodrigues Melo, técnico em Informática (cursando), IFPE, Brasi marcos.grm@yahoo.com

Clara Larissa Duarte Trajano Dias, técnica em Informática (cursando), IFPE, Brasil claralarissa0412@gmail.com

Yasmim Adrieny da Silva Sales, técnica em Informática (cursando), IFPE, Brasil yasmim.adrieny@gmail.com

Eduardo Garcia Wanderley, Msc, IFPE, Brasil eduardo.wanderley@garanhuns.ifpe.edu.br Renata Garcia Wanderley, Dra, UFPE, Brasil <renatagw@hotmail.com> 